\title{
Hepatic Artery Infusion of Doxorubicin with Hepatic Venous Drug Extraction ${ }^{1}$
}

\author{
David A. August, M.D., ${ }^{*}$ Nikhil Verma, ${ }^{*}$ James C. Andrews, M.D., $\dagger$ \\ Mary Ann Vaerten, B.S.,† And Dean E. Brenner, M.D. $\neq$ \\ Divisions of * Surgical Oncology, $\nmid$ Interventional Radiology, and $\ddagger$ Hematology/Oncology, University of Michigan, Ann Arbor, Michigan 48109-0331 \\ Presented at the Annual Meeting of the Association for Academic Surgery, Hershey, Pennsylvania, November 10-13, 1993
}

\begin{abstract}
Hepatic artery infusion (HAI) has been used to take advantage of the steep dose-response relationship characteristic of chemotherapeutic agents. Systemic toxicity, however, remains the dose limiting factor for HAI of low hepatic extraction drugs. This investigation compared the pharmacokinetics of doxorubicin administered using a system that combines HAI and hepatic venous drug extraction (HVDE) versus systemic administration without HVDE. HAI was accomplished by transfemoral cannulation of the hepatic artery. HVDE was aided by use of a double-balloon catheter inserted fluoroscopically via femoral vein cutdown into the infe-
\end{abstract} rior vena cava. Inflation of the balloons above and below the hepatic veins allowed collection of hepatic venous effluent. Hepatic venous blood was pumped through the double-balloon catheter into an extracorporeal circuit with activated carbon filters to extract drug prior to return to the systemic circulation. Domestic female swine $(25-35 \mathrm{~kg})$ received $3 \mathrm{mg} / \mathrm{kg}$ doxorubicin over $90 \mathrm{~min}$ via HAI. HVDE was performed for $240 \mathrm{~min}$ following initiation of HAI (Time 0-240 $\mathrm{min}$ ). Control swine underwent hepatic venous isolation using the double-balloon catheter without drug filtration and received $3 \mathrm{mg} / \mathrm{kg}$ doxorubicin over $90 \mathrm{~min}$ via systemic vein (SYSI). Serum and myocardial doxorubicin and doxorubicinol levels were assayed using HPLC. Blood was serially sampled from hepatic vein blood, from the extracorporeal circuit after filtration, and from a systemic artery. Area under the curve (AUC) was integrated from time-concentration plots over Time 0-180 min. During HAI, AUCs (nmole $\cdot \min$ ) in the hepatic venous effluent, in the extracorporeal circuit after filtration, and in systemic blood were $113 \pm$ $32.0,12.6 \pm 4.82$, and $16.9 \pm 8.43$, respectively. During SYSI, hepatic vein and systemic AUCs were 83.6 \pm 22.1 and $270 \pm 112$, respectively, the later significantly greater $(P=0.017)$ than that during HAI. After hepatic arterial infusion, cardiac tissue concentrations of doxorubicin and doxorubicinol were 16.8-fold less

\footnotetext{
1 This work was supported in part by a University of Michigan Rackham Grant and Delcath Systems, Inc. of Stamford, CT.
}

and $>4.0$-fold less, respectively, than those after systemic infusion. These data show that HAI with HVDE increases hepatic delivery of doxorubicin with reduced systemic and cardiac drug exposure. This method may be applicable to other drugs poorly extracted by the liver and to clinical situations in which enhanced regional drug delivery is desirable. 1994 Academic Press, Inc.

\section{INTRODUCTION}

Treatment of primary and secondary hepatic malignancies is beneficial to only a limited number of the patients suffering from these fatal conditions. Surgical resection, while potentially curative, is an appropriate option only for those few patients whose cancer is isolated to the liver, who have technically resectable disease, and whose medical condition will allow major surgery [1, 2]. Systemic chemotherapy for either primary liver tumors or hepatic metastatic disease rarely results in long-term tumor control $[3,4]$. The failure of cytotoxic therapy to control liver malignancies is due in part to the inability to administer drugs with sufficient dose intensity while avoiding potentially lethal systemic toxicity (inadequate therapeutic index).

Hepatic artery infusion chemotherapy has been used to take advantage of the steep dose-response relationship characteristic of chemotherapeutic agents [5]. Hepatic artery infusion achieves high intrahepatic drug levels and allows liver metabolism to limit systemic drug exposure. Because of their efficient metabolism by the liver, agents with high first-pass hepatic clearance (such as 5-fluorodeoxyuridine) are particularly attractive targets for hepatic artery infusion. Unfortunately, while tumor response rates to hepatic artery infusion are high, this success has never been demonstrated to result in improved clinical outcomes as hepatic toxicity is common when high extraction drugs are administered at tumoricidal doses $[6,7]$.

For low hepatic extraction drugs, systemic toxicity remains the dose-limiting obstacle to regional infusion che- 
motherapy. Methods of isolated hepatic perfusion (hepatic artery infusion with simultaneous hepatic venous drug extraction) have been developed to overcome these difficulties [8-15]. Infusion of high concentrations of antineoplastic drug into tumor-containing liver combined with removal of drug in the hepatic venous effluent before it reaches the systemic circulation potentially takes advantage of the steep dose-response curve of cancers to chemotherapy while minimizing systemic side effects. Unfortunately, only the method of isolated hepatic perfusion described by August et al. [16] and by Curley et al. [15] do not involve major operative procedures. The physiologic insult of such operative interventions has precluded the use of isolated hepatic perfusion in "sick" patients and prevented the use of drug regimes that require multiple treatment courses.

Doxorubicin, a drug with low first-pass liver metabolism, has been administered via hepatic artery infusion to treat hepatocellular carcinoma and other intrahepatic malignancies [3]. Tumor response rates to doxorubicincontaining regimens administered via the hepatic artery have been reported to be as high as 50\% [17]. An anthracycline, doxorubicin is reduced in cellular cytoplasm by aldehyde and ketone reductases and detoxified in the hepatocyte, most probably by NADPH cytochrome P-450 reductase-catalyzed reduction of the oxygen linked glycoside to deoxyglycone forms [18-21]. Doxorubicin and its primary alcohol metabolite doxorubicinol are excreted unchanged in the bile and to a lesser extent in the urine [19]. Accurate prediction of acute human clinical toxicity from doxorubicin by empiric determination of plasma pharmacokinetics of the drug or its metabolites has not yet proved feasible $[22,23]$. Nevertheless, doxorubicinol may be the primary specie responsible for toxicity observed following doxorubicin administration [24].

We have developed a model to study hepatic metabolism of doxorubicin. This model permits regional infusion of drug to the liver via the hepatic artery, collection of all hepatic venous effluent, and removal of drug from the effluent prior to systemic reinfusion. This is achieved percutaneously with the use of a specially developed double-balloon catheter which is positioned in the inferior vena cava to isolate and collect all hepatic venous blood; this method avoids the confounding pathophysiologic effects of laparotomy. This report describes the use of this model to determine the ability of hepatic artery infusion of doxorubicin combined with hepatic venous drug extraction: (a) to limit systemic plasma levels of doxorubicin following hepatic artery infusion; (b) to achieve high levels of hepatic exposure to doxorubicin while limiting systemic exposure (as assessed by hepatic and myocardial drug and metabolite levels); and (c) to increase the ratio of liver:systemic exposure to doxorubicin over that seen with systemic administration of the same drug dose.

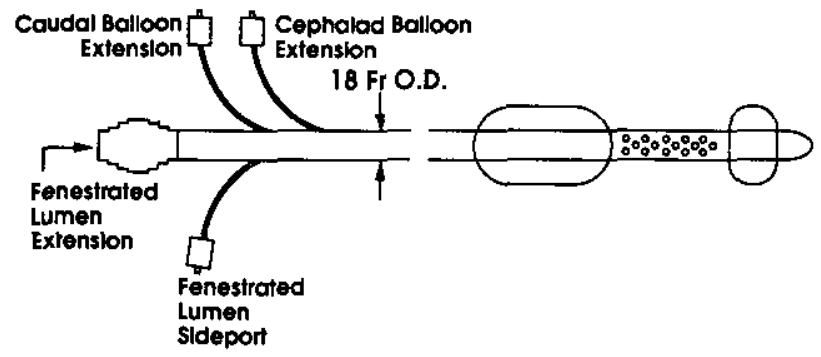

FIG. 1. The Bodden catheter. The Bodden catheter (Delcath, Inc., Stamford, CT) is a four-lumen, double-balloon, polyethylene catheter. Two lumina of the Bodden catheter are used to independently inflate and deflate caudal and cephalad balloons. Proper positioning of the Bodden catheter within the inferior vena cava allows inflation of the caudal balloon superior to the renal veins and inflation of the cephalad balloon in the suprahepatic vena cava, just below the right atrium, isolating the portion of the inferior vena cava into which the hepatic veins drain. The cephalad balloon is separated from the caudal balloon by a $6-\mathrm{cm}$ fenestrated portion of the catheter. The fenestrations are in continuity with a third, main lumen of the catheter. The main lumen allows withdrawal of hepatic venous effluent into an extracorporeal circuit. The fourth lumen of the catheter (not visible in this figure) bypasses the main lumen, allowing blood to flow from the inferior vena cava below the caudal balloon through the catheter and out the tip into the suprahepatic vena cava.

\section{MATERIALS AND METHODS}

Hepatic venous isolation/hepatic venous drug extraction. Hepatic venous isolation and hepatic venous drug extraction were accomplished using a Bodden catheter in combination with activated charcoal filtration of drug containing hepatic venous effluent. The Bodden catheter (Delcath, Inc., Stamford, CT; Fig. 1) is a four-lumen, double-balloon, polyethylene catheter designed to be inserted through the femoral vein either percutaneously (utilizing a modified Seldinger technique) or by venous cutdown. The latter method was used in these studies because of the relatively small caliber of the porcine femoral vein. Two lumina of the Bodden catheter are used to independently inflate and deflate caudal and cephalad balloons. The cephalad balloon is separated from the caudal balloon by a $6-\mathrm{cm}$ fenestrated portion of the catheter. The fenestrations are in continuity with the main lumen of the catheter. Proper positioning of the Bodden catheter within the inferior vena cava allows inflation of the caudal balloon superior to the renal veins and inflation of the cephalad balloon in the suprahepatic vena cava, just below the right atrium. This isolates all hepatic venous blood (Fig. 2), which is then withdrawn through the fenestrations and main lumen into an extracorporeal circuit. The fourth lumen of the catheter bypasses the main lumen, allowing blood to flow from the inferior vena cava below the caudal balloon through the catheter and out the tip into the suprahepatic vena cava.

Hepatic venous effluent was circulated through an extracorporeal circuit made of $\frac{1}{4}$-in. Tygon tubing by a centrifugal capacitance pump (Biomedicus Model 520 with 


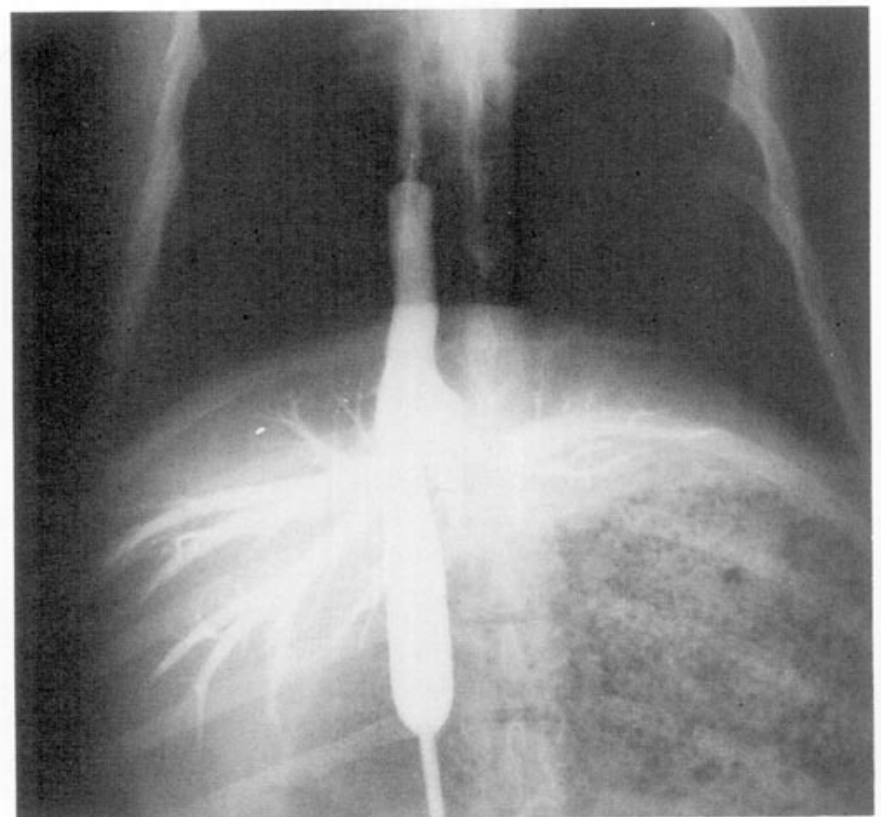

FIG. 2. Inferior vena cavagram demonstrating hepatic venous isolation by the Bodden catheter. Inferior vena cavagram obtained by retrograde injection of contrast through the main lumen of the Bodden catheter while the caudal and cephalad balloons are inflated. The contrast fills the hepatic venous tree of the pig, indicating complete capture of hepatic venous effluent. There is no evidence of contrast leaking out of the isolated segment of inferior vena cava.

a BP-50 disposable Bio-pump cartridge; Bio-Medicus, Inc., Minneapolis, MN) capable of circulating up to 10 liters of blood/min. Extracorporeal flow was monitored with an in-line flow transducer (Bioprobe Transducer Model TX20P; Biomedicus). The effluent was pumped through a pair of parallel activated carbon hemoperfusion filters (Diakart; National Medical Care Inc., Rockleigh, NJ) and then returned to the pig via an internal jugular central venous catheter (Fig. 3). The 12-French caliber venous return catheter and a 7-French carotid artery catheter used for continuous blood pressure monitoring were placed by cutdown.

\section{Swine and operative procedures.}

Six female domestic swine (Hodgin's Kennels, Howell, MI), weighing 25-35 kg, were studied. All experiments were performed in the morning following a 12 -h fast. Anesthesia was induced using $0.04 \mathrm{mg} / \mathrm{kg}$ atropine, and either $1 \mathrm{mg} / \mathrm{kg}$ ketamine and $2 \mathrm{mg} / \mathrm{kg}$ Rompun (Miles, Inc., Shawnee Mission, KS) or $4 \mathrm{mg} / \mathrm{kg}$ Telazol (Aveco Co., Inc., Fort Dodge, IA) and $2 \mathrm{mg} / \mathrm{kg}$ Rompun. General anesthesia with endotracheal intubation and spontaneous ventilation was maintained using isoflurane. Mean arterial blood pressure was continuously monitored and maintained above $65 \mathrm{~mm} \mathrm{Hg}$ primarily by infusing lactated Ringer's solution, approximately $40-50 \mathrm{cc} / \mathrm{kg} / \mathrm{hr}$. Because the activated charcoal filters absorb catecholamines from blood, epinephrine $(0.1-0.3$ $\mu \mathrm{g} / \mathrm{kg} / \mathrm{min}$ ) was also infused as needed to maintain mean arterial blood pressure.

Heparin $(200 \mathrm{U} / \mathrm{kg})$ was given by intravenous bolus hourly starting just prior to insertion of the Bodden catheter to prevent catheter and extracorporeal circuit thrombosis. For experiments requiring hepatic artery infusion of drug, an hepatic artery catheter was inserted via femoral artery cutdown and manipulated under fluoroscopic guidance into the proper hepatic artery. If the arterial anatomy prevented placement of the tip of the catheter beyond the takeoff of the gastroduodenal artery while still perfusing the entire liver, the gastroduodenal artery was angiographically embolized using a Gelfoam (Upjohn, Kalamazoo, MI) plug. The Bodden catheter was inserted by cutdown on the femoral vein and advanced into the inferior vena cava. Proper positioning was achieved fluoroscopically.

Hepatic venous isolation was achieved by inflating the caudal and cephalad balloons of the Bodden catheter prior to initiating drug infusion. The centrifugal pump rate was constantly monitored and adjusted to pump all hepatic venous effluent while preventing development of negative pressure sufficient to collapse the isolated segment of inferior vena cava or the hepatic veins.

All animals were sacrificed by lethal injection of Beuthanasia-D (Schering-Plough Animal Health, Kenilworth, $\mathrm{NJ}$ ) at completion of each study. At the time of sacrifice, anatomic autopsy was performed to assure that there was no drug extravasation and that the hepatic

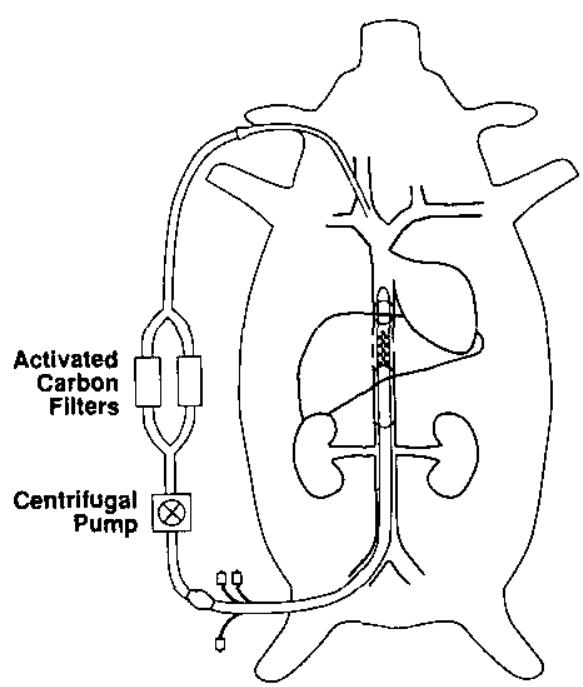

FIG. 3. Schematic representation of the extracorporeal hepatic venous drug extraction circuit. Hepatic venous effluent collected by the intracaval double-balloon catheter was circulated through an extracorporeal circuit by a centrifugal capacitance pump capable of circulating up to 10 liters of blood/min. Extracorporeal flow was monitored with an in-line flow transducer. The effluent was pumped through a pair of parallel activated carbon hemoperfusion filters and then returned to the pig via an internal jugular central venous catheter. 


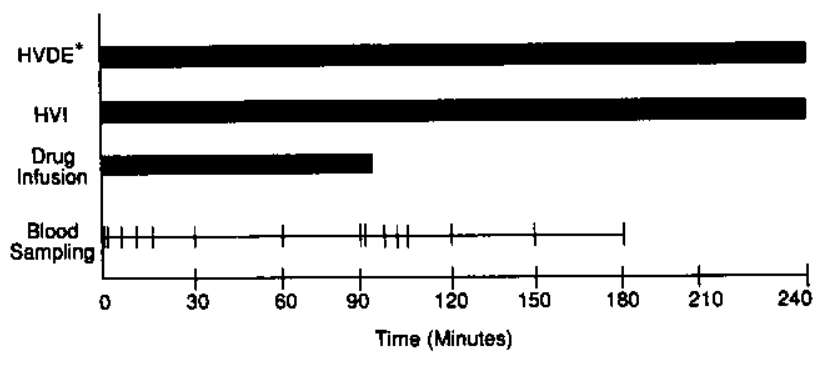

- HVDE only in pigs receiving hepatic artery drug infusion.

FIG. 4. Experimental design (see text). HVDE, hepatic venous drug extraction; HVI, hepatic venous isolation.

artery and Bodden catheters were positioned properly to truly achieve hepatic artery infusion with hepatic venous drug extraction.

These studies were approved by the Subcommittee on Animal Studies of the Ann Arbor Veterans Administration Medical Center.

Doxorubicin administration (Fig. 4). Clinical grade doxorubicin hydrochloride obtained from the University of Michigan Hospitals inpatient pharmacy was used for all studies. Drug was administered via hepatic artery infusion to three swine and via systemic internal jugular vein infusion to three swine. All animals received a total dose of $3 \mathrm{mg} / \mathrm{kg}(5.52 \mu \mathrm{mole} / \mathrm{kg})$ over $90 \mathrm{~min}$. Hepatic venous isolation with drug extraction was performed for $240 \mathrm{~min}$ following initiation of hepatic artery infusions (Time $0-240 \mathrm{~min}$ ). In swine receiving doxorubicin via systemic infusion, hepatic venous isolation for $240 \mathrm{~min}$ using the Bodden catheter was performed without hepatic venous drug extraction (the filters were omitted from the extracorporeal circuit). This permitted pharmacokinetic isolation of the liver without altering systemic distribution of the drug.

In all experiments hepatic venous (obtained from the extracorporeal circuit prior to filtration) and systemic blood samples were obtained periodically for determination of doxorubicin and doxorubicinol concentrations. In swine undergoing hepatic artery infusion/hepatic venous drug extraction, blood samples were also obtained from the extracorporeal circuit after filtration to determine the efficiency of drug filtration. Samples were obtained $0,1,5,10,15,30,60$, and 90 min following initiation of the drug infusion, and at $91,95,100,105,120,150$, and $180 \mathrm{~min}$ following initiation of the infusion (during the "washout phase"). At completion of each experiment (Time $240 \mathrm{~min}$ ), tissue samples were obtained from the heart to assay myocardial doxorubicin and doxorubicinol levels.

Drug analysis. High-pressure liquid chromatography (HPLC) grade tetrahydrofuran and certified grade ammonium formate, chloroform, and ammonium sulfate were obtained from Fisher Laboratories of Allied Industries Inc. (Pittsburgh, PA). A doxorubicinol standard was synthesized according to the published procedures of Takanashi and Bachur [25]. Purity of the doxorubicin and doxorubicinol standards was confirmed by a single peak on HPLC at published retention times [26]. The lack of other peaks in the HPLC trace at the sensitivity used suggested at least $95 \%$ purity. Specimens were assayed by HPLC after a chloroform:isopropanol $(1: 1, v / v)$ extraction according to a previously published procedure [26]. The technique was modified by the use of a $15-\mathrm{cm}$ $\mu$ Bondapak phenyl column (Waters Associates, Millipore Corp., Milford, MA).

Data analysis and pharmacokinetics. Doxorubicin and doxorubicinol plasma concentrations were calculated, stored, pharmacokinetically fit, and statistically analyzed on Excel 4.0 (Microsoft, Redmond, WA). Doxorubicin pharmacodynamics (the relationship between dose/route of administration and biologic effect) may be modeled by the equation $\mathrm{SF}=e^{-k C T}$, where $\mathrm{SF}$ equals the fraction of tumor cells killed, $C$ is the drug concentration, $T$ is the exposure time, and $k$ is a constant. The product $C T$ is equivalent to the area under the plasma time/concentration curve (AUC). Thus AUC was chosen as the parameter to quantitate hepatic and systemic drug exposure because AUC relates directly to the clinical pharmacodynamics of doxorubicin [27]. The area under the time/concentration curve was integrated by calculating the sum of the trapezoids formed by data points between Times 0 and $180 \mathrm{~min}$, interpolating missing data when necessary, using KaleidaGraph (Synergy Software, Reading, PA). Student's $t$ test was used to assess statistical significance.

\section{RESULTS}

\section{Hemodynamic Effects of Hepatic Venous Isolation}

A total of six pigs received $3 \mathrm{mg} / \mathrm{kg}$ doxorubicin over $90 \mathrm{~min}$ with simultaneous hepatic venous isolation using the double-balloon catheter. Three received drug via systemic infusion without drug extraction (SYSI group), and three received drug via hepatic artery infusion with hepatic venous drug extraction (HAI group). All animals survived to completion of the pharmacokinetic data collection at $180 \mathrm{~min}$ after initiation of the doxorubicin infusion (90 $\mathrm{min}$ after cessation of the infusion). Mean arterial pressure (MAP) ranged between 64 and $86 \mathrm{~mm}$ $\mathrm{Hg}$ and was stable throughout the period of hepatic venous isolation in both the SYSI and HAI groups (Fig. 5a). Mean MAP was similar in both groups throughout hepatic venous isolation. Mean total hepatic blood flow was measured in the extracorporeal circuit in two of the HAI and three of the SYSI pigs; it ranged between 650 and $850 \mathrm{ml} / \mathrm{min}$ and was somewhat higher in SYSI than in HAI pigs (Fig. 5b). This is likely due to the hepatic artery catheter in the HAI pigs restricting hepatic artery 

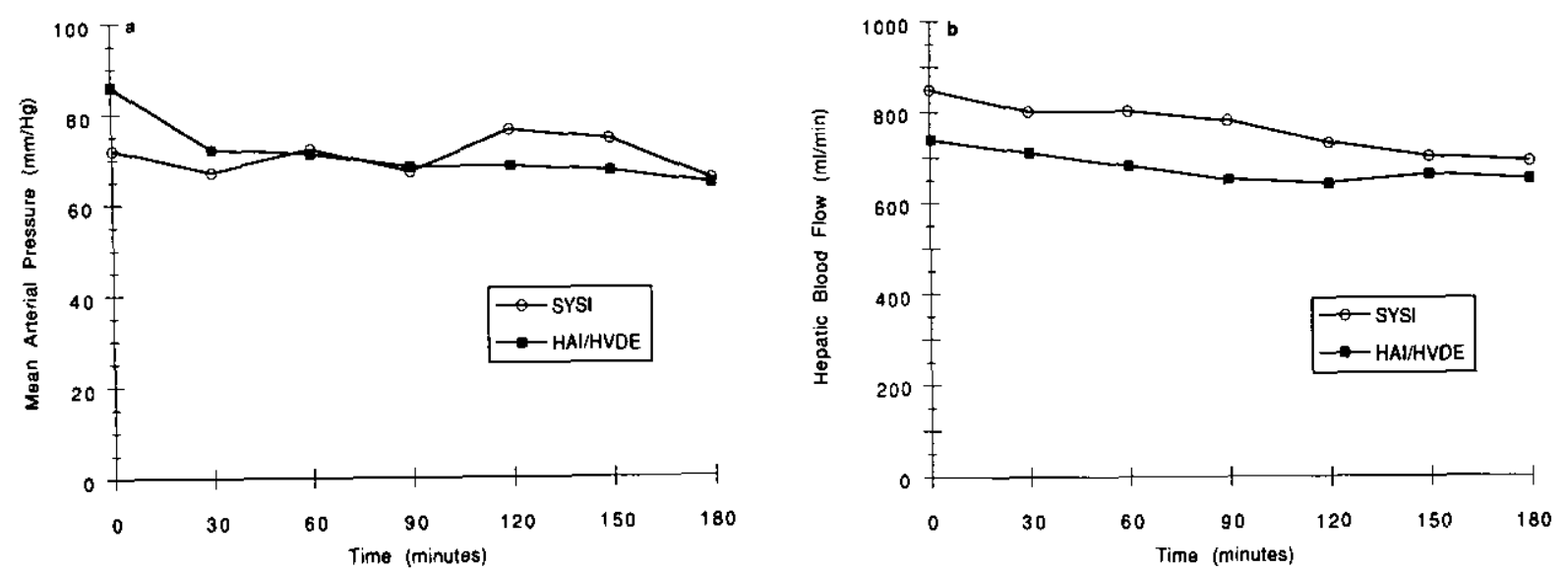

FIG. 5. Hemodynamic parameters during hepatic venous isolation. (a) Mean arterial blood pressure, as measured in the carotid artery during hepatic venous isolation, remained stable in pigs receiving SYSI or HAI/HVDE. (b) Total hepatic blood flow, as measured by a flow probe in the extracorporeal circuit, decreased minimally during hepatic venous isolation. SYSI, systemic infusion of doxorubicin; $\mathrm{HAI} / \mathrm{HVDE}$, hepatic artery infusion of doxorubicin with hepatic venous drug extraction.

flow. Mean hepatic blood flow decreased approximately $15 \%$ in both SYSI and HAI pigs during the experiments.

\section{Efficiency of Hepatic Venous Drug Extraction}

The doxorubicin extraction efficiency (EE) of the extracorporeal filters used during hepatic artery infusion was calculated using the formula $\mathrm{EE}=\left(C_{\mathrm{i}}-C_{\mathrm{o}}\right) / C_{\mathrm{i}}$, where $C_{\mathrm{i}}$ equals the concentration of doxorubicin in the blood going into the filters (hepatic venous blood) and $C_{\circ}$ is the concentration in the blood leaving the filters in the extracorporeal circuit. Mean filter extraction efficiency in the three HAI pigs ranged from 0.82 to 0.92 during the duration of the experiments (Fig. 6).

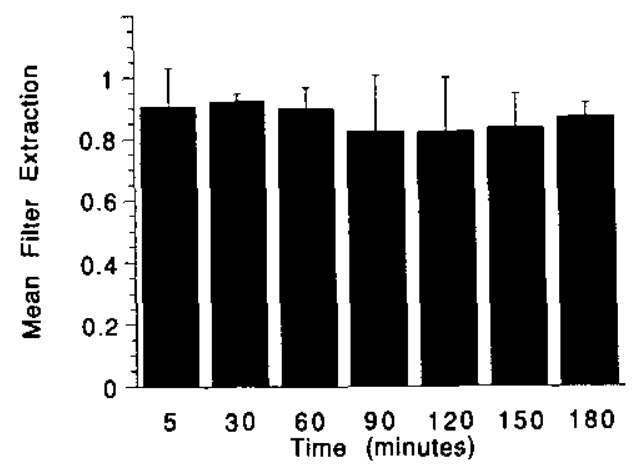

FIG. 6. Efficiency of filter extraction of doxorubicin during hepatic artery infusion/hepatic venous drug extraction. Doxorubicin extraction efficiency (EE) of the extracorporeal hepatic venous drug extraction filters used during hepatic artery drug infusion was calculated using the formula $\mathrm{EE}=\left(C_{\mathrm{i}}-C_{\mathrm{o}}\right) / C_{\mathrm{i}}$, where $C_{\mathrm{i}}$ equals the concentration of doxorubicin in the blood going into the filters (hepatic venous blood) and $C_{\mathrm{o}}$ is the concentration of doxorubicin in the blood leaving the filters. Doxorubicin $(3 \mathrm{mg} / \mathrm{kg})$ was infused through an hepatic artery catheter over $90 \mathrm{~min}$; hepatic venous drug extraction, initiated at the beginning of the drug infusion, was maintained for a total of 240 min. Extraction efficiencies graphed are means from three pigs; error bars represent standard deviations.

\section{Hepatic Venous and Systemic Circulation Drug Exposure}

During hepatic artery infusion of doxorubicin, systemic plasma concentrations of drug at all times were 2.5- to 10 -fold lower than those observed in the hepatic venous effluent (Fig. 7a). During SYSI, systemic doxorubicin plasma concentrations were always greater than those observed in the hepatic venous effluent, by a factor of 2.5-30. To determine the relative hepatic venous and systemic plasma exposures to doxorubicin during the drug infusions, the relevant AUCs were computed for each pig and then the group means were calculated (Table 1). Systemic plasma exposure to doxorubicin in SYSI pigs was 16.0 times greater than that in the $\mathrm{HAI}$ pigs receiving the same $3 \mathrm{mg} / \mathrm{kg}(5.52 \mu \mathrm{M} / \mathrm{kg})$ dose $(P=0.017)$.

\section{Myocardial Doxorubicin and Doxorubicinol Levels}

At Time $240 \mathrm{~min}$ all pigs were sacrificed and tissue samples were obtained from the apex of the heart for measurement of doxorubicin and doxorubicinol levels (Table 2). After hepatic arterial infusion, cardiac tissue concentrations of doxorubicin and doxorubicinol were 16.8-fold and more than 4.0-fold less, respectively, than those after systemic infusion. As hepatic vein AUCs were similar with both routes of administration, these data suggest achievement of at least a 4-fold decrease in potential cardiac toxicity associated with hepatic artery infusion while attaining similar hepatic therapeutic exposure. The factor of four may be an underestimate as additional doxorubicin measured in the myocardium would have been converted to toxic doxorubicinol if the pigs had not been sacrificed after $240 \mathrm{~min}$.

\section{DISCUSSION}

Regional infusion of cancer chemotherapy offers two theoretical advantages over systemic administration. 

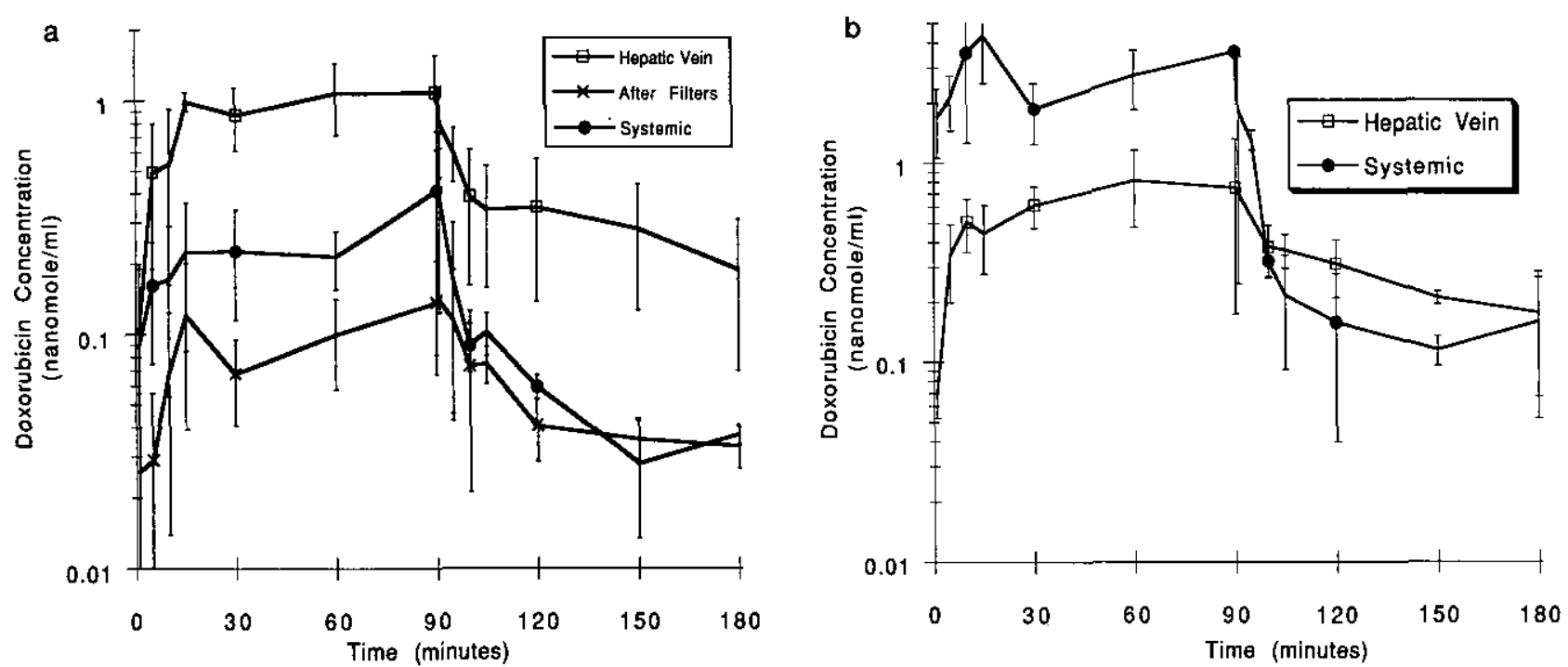

FIG. 7. Time/concentration curves for doxorubicin sampled at various locations. Time/concentration curves were plotted for plasma sampled from systemic blood and hepatic venous blood during HAI/HVDE and during SYSI of doxorubicin. Blood was also sampled from the extracorporeal circuit after filtration in pigs undergoing HAI/HVDE. All infusions were doxorubicin $(3 \mathrm{mg} / \mathrm{kg})$ administered over $90 \mathrm{~min}$. Hepatic venous isolation and HVDE were continued until sacrifice of the pigs 240 min after initiation of the drug infusion. Curves were plotted from mean values in three pigs in each group; error bars are standard deviations. (a) Time/concentration curve during HAI/HVDE; (b) time/concentration curve during SYSI. SYSI, systemic infusion of doxorubicin; HAI/HVDE, hepatic artery infusion of doxorubicin with hepatic venous drug extraction.

First, regional infusion can increase local delivery of drug and thereby exploit the steep dose-response relationship characteristic of many cytotoxic drugs. Second, toxicity may be reduced because regional metabolism of drug can decrease systemic exposure [28, 29]. The liver has been a particularly promising target for regional infusion of chemotherapy via the hepatic artery because of the frequency of primary and metastatic hepatic malig-

TABLE 1

\begin{tabular}{lccc} 
& Mean AUCs \\
\hline & \multicolumn{3}{c}{ AUC $^{a}(\mathrm{nmole} \cdot \mathrm{min} / \mathrm{ml})$} \\
\cline { 2 - 4 } \multicolumn{1}{c}{ Infusion } & Hepatic vein & After filters & Systemic \\
\hline HAI/HVDE $^{b}$ & $113 \pm 32.0$ & $12.6 \pm 4.82$ & $16.9 \pm 8.43^{*}$ \\
SYSI $^{c}$ & $83.6 \pm 22.1$ & - & $270 \pm 112^{*}$ \\
\hline
\end{tabular}

Note. Area under the time/concentration curve was computed from doxorubicin concentrations measured sequentially in hepatic vein plasma (sampled from the extracorporeal circuit before filtration), after filters plasma (sampled from the extracorporeal circuit after filtration in pigs receiving hepatic artery infusion only), and systemic plasma (sampled from the carotid artery). Numbers represent the mean ( \pm standard deviation) AUCs from three pigs that received hepatic artery infusion of doxorubicin ( $3 \mathrm{mg} / \mathrm{kg}$ over $90 \mathrm{~min}$ ) and three pigs that received systemic infusion of doxorubicin $(3 \mathrm{mg} / \mathrm{kg}$ over 90 $\min$ ).

${ }^{a}$ Area under time/concentration curve.

${ }^{b}$ Hepatic artery infusion with hepatic venous drug extraction.

${ }^{c}$ Systemic infusion.

${ }^{*} P=0.017$. nancies, because (particularly for tumors of primary gastrointestinal or hepatic origin) the liver may be the sole site of tumor but technical factors preclude surgical resection [1], and because the hepatic artery is the predominant source of tumor blood flow, whereas the portal vein is the main source of blood flow to normal liver parenchyma $[29,30]$.

There has been considerable interest in the administration of doxorubicin via hepatic artery infusion because of preliminary experiences demonstrating significant response rates of hepatocellular carcinomas [31,32] and metastatic neuroendocrine tumors [33]. Unfortunately, the pharmacokinetic advantages of hepatic artery infusion of doxorubicin seem to be limited. In a rab-

TABLE 2

Mean Tissue Concentrations (nmole/gm of tissue) of Doxorubicin and Doxorubicinol

\begin{tabular}{lccc}
\hline Compound & HAI/HVDE $^{a}$ & \multicolumn{1}{c}{ SYSI $^{b}$} & Ratio SYSI:HAI \\
\hline DOX $^{c}$ & $0.44 \pm 0.20$ & $7.39 \pm 4.21$ & 16.8 \\
DOXOL $^{d}$ & $<0.001$ & $0.004 \pm 0.007$ & $>4$ \\
\hline
\end{tabular}

Note. Tissue samples were obtained from the apex of the heart at completion of each experiment ( 240 minutes after initiation of a 90 minute infusion of doxorubicin, $3 \mathrm{mg} / \mathrm{kg}$ ). Numbers represent mean ( \pm standard deviation).

${ }^{a}$ Hepatic artery infusion with hepatic venous drug extraction.

${ }^{b}$ Systemic infusion.

${ }^{c}$ Doxorubicin.

${ }^{d}$ Doxorubicinol. 
bit model, maximum serum concentrations and heart and liver tissue concentrations of doxorubicin and doxorubicinol were similar following either systemic or hepatic arterial infusion; systemic doxorubicin AUC was likewise unaffected by route of administration [34]. Interestingly, in this short-term infusion (drug given over $5 \mathrm{~min}$ ) model, liver tumor doxorubicin levels were higher following hepatic artery infusion, suggesting potential therapeutic enhancement. In humans, prolonged hepatic artery infusion of doxorubicin to treat hepatocellular carcinoma can cause dose related cardiac toxicity, alopecia, and myelosuppression as is seen following systemic administration.

The current study was undertaken to determine whether the pharmacokinetic parameters of doxorubicin distribution and metabolism could be altered such that the theoretical advantages of hepatic artery infusion could be realized. A method of hepatic venous isolation combined with hepatic venous drug extraction was used in an attempt to increase the pharmacokinetic efficacy of hepatic artery doxorubicin administration. The parameters measured to assess the utility of this method were: (a) comparison of plasma levels of doxorubicin following systemic administration or regional hepatic administration with hepatic venous drug extraction; (b) comparison of plasma AUCs of doxorubicin following systemic administration or regional hepatic administration with drug extraction; and (c) comparison of cardiac exposure to doxorubicin (as assessed by myocardial doxorubicin and doxorubicinol tissue levels) following hepatic artery infusion with hepatic venous drug extraction and systemic infusion. Plasma AUC was chosen as the primary measure of hepatic and systemic exposure to doxorubicin because doxorubicin pharmacodynamics (the relationship between dose/route of administration and biologic effect) may be modeled by the equation SF $=e^{-k C T}$, where SF equals the fraction of tumor cells killed, $C$ is the drug concentration, $T$ is the exposure time, and $k$ is a constant. The product $C T$ is equivalent to the AUC. Thus, AUC should relate directly to the therapeutic effect of a given dose and route of doxorubicin administration [27]. Tissue levels of doxorubicin and doxorubicinol were monitored because of their relationships to therapeutic efficacy and toxicity respectively. From the pharmacodynamic argument made above, increased tissue levels of doxorubicin should correlate directly with improved cytotoxicity. Doxorubicinol is an important metabolic product of doxorubicin. It is less cytotoxic in vitro [35-37] than doxorubicin; however, in a model of papillary muscle function and a model of isolated ion channels, doxorubicinol is 5 - to 50 -fold more toxic $[38,39]$. Thus tissue levels of doxorubicinol may correlate with a major dose-limiting toxicity of doxorubicin, dose-dependent cardiomyopathy.

By all three of these criteria, the method of hepatic artery infusion/hepatic venous drug extraction tested in this study improved the pharmacokinetic efficacy of doxorubicin in comparison with systemic administration of an identical dose. Although peak hepatic vein concentrations of doxorubicin were similar following both hepatic artery and systemic infusion of doxorubicin (1.08 and $0.75 \mathrm{nmole} / \mathrm{ml}$, respectively), peak systemic concentration of doxorubicin was lower following hepatic artery infusion $(0.41 \mathrm{nmole} / \mathrm{ml})$ than after systemic infusion ( $3.6 \mathrm{nmole} / \mathrm{ml}$ ) (Fig. 7). Furthermore, systemic exposure to doxorubicin as assessed by AUC was statistically significantly less following hepatic artery infusion than after systemic infusion by a factor of 16.0 while achieving similar hepatic exposure as assessed by hepatic vein AUC (Table 1). Finally, tissue drug levels suggest decreased potential systemic toxicity with hepatic artery infusion. Although not statistically significant, myocardial concentrations of both doxorubicin and doxorubicinol were less after hepatic artery infusion than after systemic infusion (Table 2).

The improved pharmacokinetic profile associated with combining hepatic artery infusion of doxorubicin with hepatic venous drug extraction is apparently a result of both native hepatic extraction of drug and augmentation of the inherently low native hepatic extraction of doxorubicin by hepatic venous drug extraction in the extracorporeal filters. The effect of native liver extraction of doxorubicin is evident in Fig. $7 \mathrm{~b}$, where it is observed that hepatic vein drug levels in pigs receiving systemic infusions were consistently lower than drug levels in the systemic circulation, resulting in a 3.2 -fold reduction in AUC in the hepatic vein (Table 1). Augmentation of this native extraction by the extracorporeal filters is evident from the 9.0 -fold reduction after filter drug exposure compared to hepatic vein doxorubicin exposure as assessed by AUCs in the hepatic artery infusion/hepatic venous drug extraction pigs (Table 1). The relative contributions of each of these factors (change in route of drug administration versus filter extraction) could be assessed by repeating these experiments using an additional control group that receives doxorubicin via hepatic artery infusion but without hepatic venous drug extraction; we have not yet studied such a control group. Preliminary results from subsequent experiments we have conducted suggest that during hepatic artery infusion liver extraction of doxorubicin is approximately $65 \%$, and after completion of the infusion there is net washout of unmetabolized doxorubicin from liver reservoirs of drug (as evidenced by net negative hepatic extraction during the washout phase). The greater than $80 \%$ extraction efficiency of the activated carbon filters in the drug extraction portion of the extracorporeal circuit (Fig. 6) limits systemic exposure to doxorubicin.

The pharmacokinetic enhancement of the therapeutic index of doxorubicin administered by hepatic artery infusion was achieved with a system that caused only minimal hemodynamic changes (Fig. 5). Furthermore, unlike other methods of hepatic vascular isolation and he- 
patic venous drug extraction [8-14], this method does not require a laparotomy, or even (in humans) general anesthesia. Thus, it may be used in patients who are "sick" because of their liver tumors or because of the effects of prior therapy. It may also be used repeatedly in the same patient, a feature not offered by methods requiring laparotomy.

These results confirm and extend findings recently reported by Curley et al. [15]. In survival experiments with a similar system in swine using 10-min infusions of doxorubicin, they found significant enhancement of hepatic extraction of doxorubicin with the use of the Bodden catheter to facilitate hepatic venous drug extraction. They did not make direct comparisons between plasma and tissue levels resulting from hepatic artery infusion with hepatic venous drug extraction and those resulting from systemic drug infusion, nor did they analyze AUCs.

In summary, the data presented demonstrate that hepatic artery infusion of doxorubicin combined with hepatic venous isolation and hepatic venous drug extraction increased hepatic delivery of doxorubicin while reducing systemic plasma and myocardial tissue exposure to doxorubicin and (in the heart) the cardiotoxic metabolite doxorubicinol. This method may be applicable to other drugs poorly extracted by the liver. Furthermore, this method may potentially be generalized to other clinical and anatomic situations in which enhanced regional drug delivery is desirable.

\section{REFERENCES}

1. August, D. A., Ottow, R. T., and Sugarbaker, P. H. Clinical perspective of human colon cancer metastasis. Cancer Metastasis Rev. 3: 303, 1984.

2. Ottow, R. T., August, D. A., and Sugarbaker, P. H. Surgical therapy of hepatobiliary tumors. In J. C. Bottino, R. Opfell, and F. Muggia (Eds.), Therapy of Tumors Confined to the Liver and Biliary Tract. Amsterdam: Martinus-Nijhoff, 1985.

3. Lotze, M. T., Flickinger, J. C., and Carr, B. I. Hepatobiliary Neoplasms. In V. T. Devita, S. Hellman, and S. A. Rosenberg (Eds.), Cancer: Principles and Practice of Oncology. Philadelphia: Lippincott, 1989. Pp. 883-914.

4. Niederhuber, J. E., and Ensminger, W. D. Treatment of metastatic cancer to the liver. In V. T. Devita, S. Hellman, and S. A. Rosenberg (Eds): Cancer: Principles and Practice of Oncology. Philadelphia: Lippincott, 1989. Pp. 2201-2224.

5. Skipper, H. E., Schabel, F. M., Mellett, L. B., et al. Implications of biochemical, cytokinetic, pharmacologic, and toxicologic relationships in the design of otimal therapeutic schedules. Cancer Chemother. Rep. 54: 431, 1970.

6. Hohn, D. C., Melnick, J., Stagg, R., et al. Biliary sclerosis in patients receiving hepatic arterial infusions of floxuridine. $J$. Clin. Oncol. 3: 98, 1985.

7. Chang, A. E., Schneider, P. D., Sugarbaker, P. H., et al. A prospective, randomized trial of regional versus systemic continuous 5 fluorodeoxyuridine chemotherapy in the treatment of colorectal liver metastases. Ann. Surg. 206: 685, 1987.

8. Kestens, P. J., Farrelly, J. A., and McDermott W. V. A technique of isolation and perfusion of the canine liver. J. Surg. Res. 1(1): $58,1961$.

9. Skibba, J. L., Almagro, U. A., Condon, R. E., and Petroff, R. J. A technique for isolation perfusion of the canine liver with survival. J. Surg. Res. 34(2): 123, 1983.

10. van de Velde, C. J. H., Kothuis, B. J. L., Barenbrug, H. W. M., et al. A successful technique of in vivo isolated liver perfusion in pigs. J. Surg. Res. 41(6): 593, 1986.

11. Ku, Y., Saitoh, M., Nishiyama, H., et al. Extracorporeal removal of anticancer drugs in hepatic artery infusion: The effect of direct hemoperfusion combined with venovenous bypass. Surgery 107: 273, 1990.

12. Schwemmle, K., and Aigner, K. Requirements and results of liver perfusion. Recent Results Cancer Res. 100: 229, 1986.

13. Aigner, K. R., Walther, H., and Link, K. H. Isolated liver perfusion with MMC/5-FU: Surgical technique, pharmacokinetics, and clinical results. Contrib. Oncol. 29: 229, 1988.

14. de Brauw, L. M., Marinelli, A., van de Velde, C. J. H., Hermans, J., Tjaden, U. R., Erkelens, C., and de Bruijn, E. A. Pharmacological evaluation of experimental isolated liver perfusion and hepatic artery infusion with 5-fluorouracil. Cancer Res. 51: 1694, 1991.

15. Curley, S. A., Byrd, D. R., Newman, R. A., et al. Reduction of systemic drug exposure after hepatic arterial infusion of doxorubicin with complete hepatic venous isolation and extracorporeal chemofiltration. Surgery 114: 579, 1993.

16. August, D. A., Bodden, W. L., Setaro, J., and Ellis, H. Percutaneous hepatic vascular isolation for regional chemotherapy. Proc. Am. Assoc. Cancer Res. 31: 427, 1990. [Abstract]

17. Carr, B. I., Starzl, T. E., Iwatsuki, S., et al. Aggressive treatment for advanced hepatocellular carcinoma (HCC): High response rates and prolonged survival. Hepatology 14: 243, 1991.

18. Bachur, N. R. Adriamycin pharmacology. Cancer Chemother. Rep. 6: 153, 1975.

19. Benjamin, R. A., Riggs, C. E., and Bachur, N. R. Plasma pharmacokinetics of Adriamycin and its metabolites in humans with normal hepatic and renal function. Cancer Res. 37: 1416, 1977.

20. Felsted, R. L., and Bachur, N. R. Mammalian carbonyl reductases. Drug Metab. Rev. 11: 1, 1980.

21. Gutierrez, P. L., Gee, M. V., and Bachur, N. R. Kinetics of anthracycline antibodies free radical formation and reductive glycosidase activity. Arch. Biochem. Biophys. 223: 68, 1983.

22. Brenner, D. E., Wiernik, P. H., Wesley, M., and Bachur, N. R. Doxorubicin dosing: Relationship to pretreatment liver function, response, and pharmacokinetics in patients with acute non-lymphocytic leukemia. Cancer 53: 1042, 1984.

23. Brenner, D. E. Approaches to the problem of individual doxorubicin dosing schedules. Pathol. Biol. 35: 31, 1987.

24. Brenner, D. E., Anthony, L. B., Halter, S., Lindsay-Harris, N., Collins, J. C., and Hande, K. R. Effect of allyl alcohol-induced sublethal hepatic damage upon doxorubicin metabolism and toxicity in the rabbit. Cancer Res. 47: 3259, 1987.

25. Takanashi, S., and Bachur, N. R. Adriamycin metabolism in man: Evidence from urinary metabolites. Drug Metab. Dispos. 4: $79,1976$.

26. Brenner, D. E., Galloway, S., Noone, R., and Hande, K. R. Improved high performance liquid chromatography assay of doxorubicin: Comparison to thin layer chromatography. Cancer Che mother. Pharmacol. 14: 139, 1985.

27. Ratain, M. J., Schilsky, R. L., Conley, B. A., and Egorin, M. J. Pharmacodynamics in cancer therapy. J. Clin. Oncol. 8: 1739, 1990.

28. Chen, H. S. G., and Gross, J. F. Intraarterial infusion of anticancer drugs: Theoretical aspects of drug delivery and review of response. Cancer Treat. Rep. 64: 31, 1980.

29. Ensminger, W. D., and Gyves, J. W. Regional cancer chemotherapy. Cancer Treat. Rep. 68: 101, 1984.

30. Ridge, J. A., Bading, J. R., Gelbard, A., Benua, R. S., and Daly, J. 
Perfusion of colorectal hepatic metastases: Relative distribution of flow from the hepatic artery and portal vein. Cancer 59: 1547, 1987.

31. Urist, M. M., and Balch, C. M. Intra-arterial chemotherapy for hepatoma using adriamycin administered via an implantable constant infusion pump. Proc. Am. Assoc. Cancer Res. 24: 148, 1990. [Abstract]

32. Bern, M. M., McDermott, W., Cady, B., et al. Intrahepatic-arterial infusion and intravenous Adriamycin for treatment of hepatocellular carcinoma. Cancer 42: 399, 1978.

33. Ruszniewski, P., Rougier, P., Roche, A., et al. Hepatic arterial chemoembolization in patients with metastases of endocrine tumors. Cancer 71: 2624, 1993.

34. Munck, J.-N., Riggi, M., Rougier, P., et al. Pharmacokinetic and pharmacodynamic advantages of pirarubicin over Adriamycin after intraarterial hepatic administration in the rabbit VX2 tumor model. Cancer Res. 53: 1550, 1993.

35. Dessypris, E. N., Brenner, D. E., Baer, M., and Hande, K. R.
Toxicity of adriamycin metabolites to human marrow erythroid and myeloid progenitors in vitro. Cancer Treat. Rep. 70: 487, 1986.

36. Dessypris, E. N., Brenner, D. E., Baer, M., and Hande, K. R. Uptake and intracellular distribution of doxorubicin metabolites in B-lymphocytes of chronic lymphocytic leukemia. Cancer Res. 48: $503,1988$.

37. Chang, B. K., Brenner, D. E., and Gutman, R. Cellular pharmacology of doxorubicinol alone and combined with verapamil in pancreatic cancer cell lines. Anticancer Res. 9: 341, 1989.

38. Boucek, R. J., Olson, R. D., Brenner, D. E., Ogunbunmi, E. M., Inui, M., and Fleischer, S. The major metabolite of doxorubicin is a potent inhibitor of membrane associated ion pumps: A correlative study on isolated membranes and cardiac muscle. J. Biol. Chem. 262: 15851, 1987.

39. Olson, R. D., Mushlin, P. S., Brenner, D. E., et al. Doxorubicin cardiotoxicity may be caused by its metabolite, doxorubicinol. Proc. Natl. Acad. Sci. USA 85: 3585, 1988. 